\title{
WA6-3 10:40 \\ Binary Excitation Based System Identification for \\ Precision Ballscrew Table
}

Pai-Yi Huang and Yung-Yaw Chen*

Keywords: system identification, binary excitation, asymmetric Coulomb friction, ballscrew table

\section{Abstract}

Mechanical devices usually come with undesirable nonlinearities such as frictions, backlashes and saturation

Under the assumption of linear systems, the commonly seen identification schemes utilize simusoidal excitation signals for parameter identifications. However, the data for identification are unavoidably distorted by the forementioned nonlinearities and the identification result is not satisfactory. In this paper, a method based on binary excitation signals is proposed. The method does not suffer from the problem of nonlinear distortions in signal shape and is able to determine the bias term for asymmetric frictions such that an accurate model can be derived. $A$ $0.01 \mu \mathrm{m}$ high precision ballscrew table with asymmetric frictions is utilized as a test plant for this approach. The result proves to be very successful.
\end{abstract}

\section{Introduction}

Proper system identification is usually the key to successful control system design. More accurate of a plant model, easier of a controller design can be. A survey of a number of friction compensation methods has recently appeared [5]. The friction models are all focus on friction characteristics.

In frequency response analysis based system identification [1], the sinusoidal-based excitation signal is injected into the plant and the corresponding output is measured. Under linear system assumption, the frequency contents for both the excitation signal and the output signal are equal. Only the magnitudes and the phases of the output signal are affected. By plotting the frequency in $\mathrm{x}$-axis and magnitudes (phases) in y-axis, the Bode plot are constructed. Using some curve-fitting tecluniques, the s-domain system model can then be extracted.

Unfortunately, the above described system identification procedures are suitable for only linear cases. The excitation signals are easily distorted when system has some nonlinearities. Furthermore, the frequency contents for excitation and measured output are not the same after the distortion. As a result, the calculated model of the system is incorrect.

Through the studies of the nonlinearity in the ballscrew-type table, the paper developed a solution for system identification under friction nonlinearity commonly existed in many mechanical systems. Binary-type $(1,-1)$ excitations are in place of sinusoidal-typc excitations for excitation signals in system identifications. With some developed algorithms, the proposed system identification method is able to find the linear part of the system model and the asymmetric Coulomb friction.

\section{The Friction Characteristics of The Ballscrew Table}

\section{A. Friction Characteristics}

The most significant nonlinearity of the ballscrew table system is friction. They are generated from sliding motion between steel balls and screw lead, and between the moving plates and its' guides. While moving at a tiny distance, e.g. $\mu m$ level, the moving speed is usually very slow. The friction forces, at this moment, dominate the characteristics of the system.

A simple experiment is conducted for showing the

*Corresponding Author: Prof. Yung-Yaw Chen, Room 345, Dept. of Electrical Engineering, National Taiwan University, Taipei, Taiwan, R.O.C., Phone \& Fax: 886-2-23660640, Email ychenicicc.ce.nilu.cdu.tw 
friction effects. The experiment is taken on the ballscrew table. With control loop opened, a $1 \mathrm{~Hz}$ frequency, 1000 D/A-Inits (with respect to 4.88 Volt) magnitude pure simusoidal velocity command signal is injected into the system, figure 1. The system output position signals are then obtained immediately from the laser scale encoder on every sampled time. The results are shown in figure 1 , and the velocities are in Figure 2.
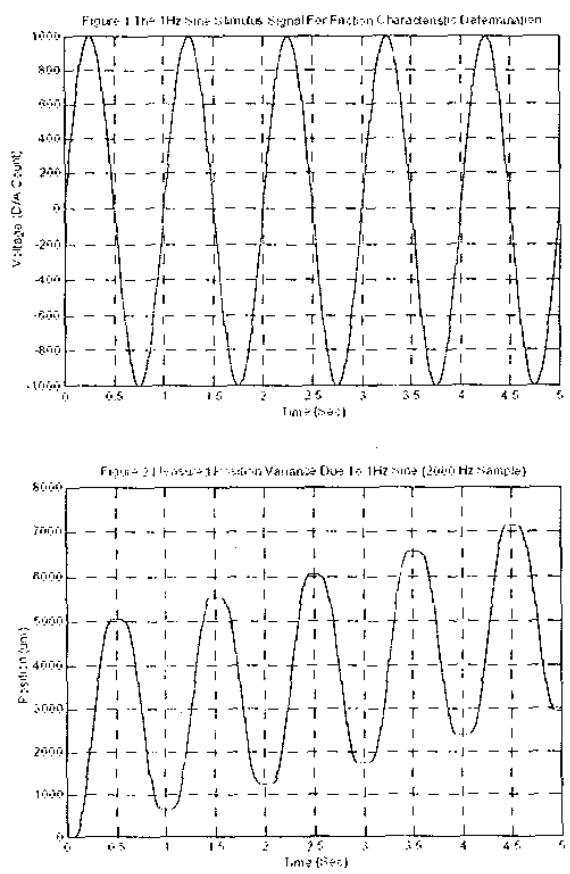

Figure 1. Velocity Command and the respective position output.

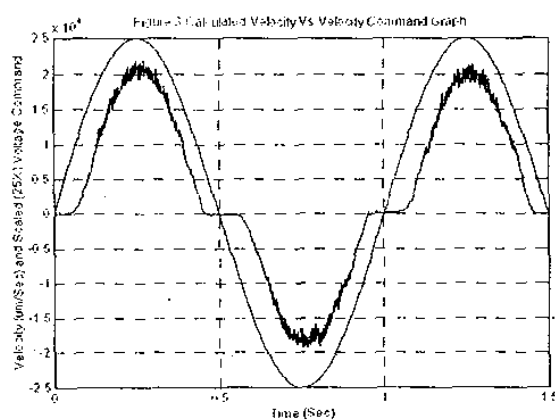

Figure 2. Velocity command Vs. Calculated Velocity
Notice that in the positive direction the friction is slight smaller than the negative one. From figure 1 , the measured positions under sinusoidal excitation are tending to drift in one direction. This is because the friction inferences are different in directions. For figure 2, around zero velocity, the static friction dominates and the controlled object will not move until the output force overcome the friction force.

\section{B. Friction model}

The friction model studied in this paper is focused on the asymmetric Coulomb friction. The formulation of the friction can be written in the following,

friction $=\left\{\begin{array}{lr}F_{c}, & v>0 \\ -F_{c}-F_{a s y m}, & v<0\end{array} \quad F_{c}>0, F_{a s m m}>0(1)\right.$

where $v$ is the velocity of the system, $F_{r}$ is the Coulomb friction and $F_{o s s m}$ is the asymmetric component of the Coulomb friction. In the case for the ballscrew table under study, the friction force is smaller in the positive direction. The asymmetric component is placed in the negative direction for convenience. All the derivations and formulations below will also apply when asymmetric component is in the positive direction.

\section{Motivations}

Because the linear part of the system model is hiding behind the nonlinearity, The friction nonlinearity increases the difficulty to identify the system. In this section, the motivations of this paper are firstly introduced. A good choice of excitation signal will help to pass through the nonlinearity and extract the exact linear plant out. Also a closed-loop filter is designed according to the asymmetric friction. At last, the identification procedures are reformulated.

The motivations of the proposed method are depicted in Figure 3. For traditional sinusoidal-based excitation signal, the waveform will be distorted when passing through the nonlinear block. The waveform will be clipped 
around the zero crossing point. Therefore, the frequency contents entering into the linear part of the system are quite different with the original desired one. As a result, the linear model calculated from the distorted output is not correct.

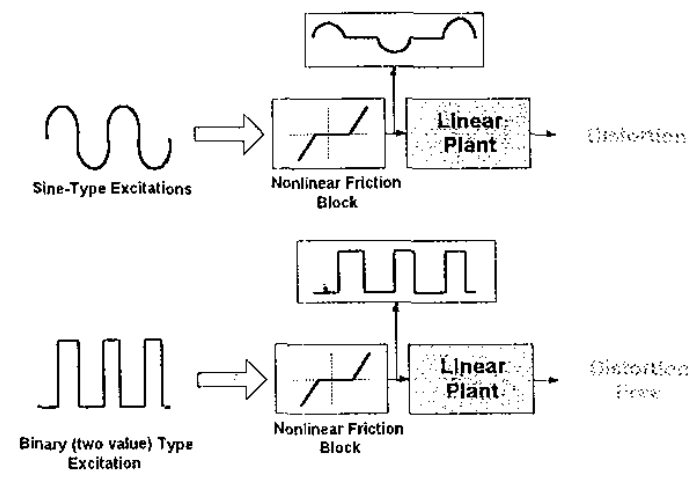

Figure 3. Motivations for choosing binary-type excitations.

If the injected signal is changed to binary-based excitation, only the magnitude of the binary signal will be affected after passing through the nonlinear block. Because of the unchanged waveform shape, the frequency contents are well reserved. The only difference is the DC part gain introduced by asymmetric friction characteristics.

The desired binary multi-frequency signal can be generated by [3]. The designed binary signal can then pass through the nonlinear block, and conduct the frequencyresponse test faithfully.

\section{Identification Procedures}

The poles and zeros of the linear system model can be evaluated exactly, but the gain of the model is not correct at this stage. The followings are the solutions for solving the problem caused by the nonlinear friction.

\section{A. Asymmetric friction determination}

As discussed in previous section, The friction characteristics of ballscrew table are asymmetric in nature. This will cause the table to drift away from the operating point especially when the testing signal is long. The difficulties for collecting experimental data are increased.

A method for deciding the asymmetric friction is developed. From experiences, the system model of the ballscrew table is in the following form,

$$
\frac{y}{u}=\frac{k}{s(s+a)\left(s^{2}+2 \xi \omega_{n} s+\omega_{n}^{2}\right)},
$$

$v$ is the system position output and $u$ is the voltage input. The mechanical induced two slower conjugate poles that dominate the system behavior accompany with a electrical produced faster pole. By ignoring the faster one, the system can be reduced to

$$
\left.\frac{y}{u}=\frac{K}{s\left(s^{2}+2 \xi \omega_{n} s+\omega_{n}^{2}\right.}\right)
$$

(a)

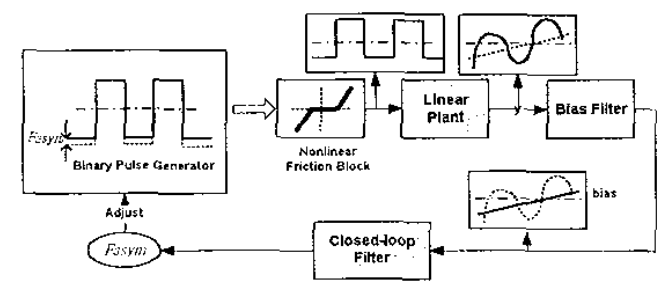

(b)

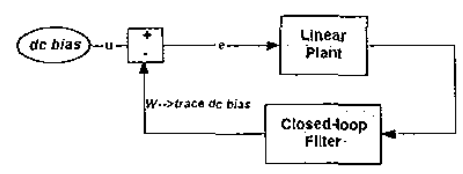

Figure 4. The closed-loop system block for finding the asymmetric component of the friction.

While applying a $50 \%$ duty-cycle-binary $(1,-1)$ signal to the input, the system output is an oscillatory signal with a varying bias. Observing from system equation (3), the system has an integrator at the denominator. When $(1,-1)$ pulse signal enter the system, it will not generate any bias in normal condition. It is the asymmetric friction block that generates the bias.

For finding the asymmetric friction, a closed-loop system is established according to Figure 4 a. The basic idea is to construct an automatic binary pulse generator that will adjust the magnitude in order to eliminate the bias. When 
the output curve of the linear plant goes to the plus direction, the force in the minus direction is then too weak and has to be increased. The output of the linear plant is rather oscillatory and it is not proper to feedback the signal directly, a low-pass filter, bias filter, is added.

$y_{\text {bias }}=\frac{\max \left(y_{-N+1} \ldots y_{0}\right)+\min \left(y_{-N+1} \ldots y_{0}\right)}{2}(4)$

In the system of Figure $4 a$, The function of the closed-loop filter is to adjust the magnitudes of the binary pulse generator according to the bias of the plant output. If the frequency of the binary pulse generator is high enough, and the low-pass frequency of the bias filter is low enough. From viewpoint of the closed-loop filter, the system in Figure $4 \mathrm{a}$ is similar to system in Figure $4 \mathrm{~b}$. The dc-bias input represents the effect of the asymmetric friction.

The key point for closed-loop filter design is letting the transfer function from bc-bias input to input of the linear plant be zero. That is

$$
\frac{e}{u}=0
$$

for this situation, the output of the closed-loop filter is equal to the asymmetric friction component that need to be found.

If the transfer function of the closed-loop filter is $\frac{n(s)}{d(s)}$, and linear plant is as in (3). The transfer function (5) of Figure $4 b$ is the following

$$
\frac{e}{u}=\frac{s\left(s^{2}+2 \xi \omega_{n} s+\omega_{n}^{2}\right) d(s)}{s\left(s^{2}+2 \xi \omega_{n} s+\omega_{n}^{2}\right) d(s)+K \cdot n(s)} .
$$

According the final value Theorem [1] and considering the stability of the denominator roots in (6), a KI type filter is chosen and it is enough for all the requirements. The closed-loop filter is the case of ballscrew table is

$$
\frac{n(s)}{d(s)}=\frac{k_{1} s+b}{s}
$$

\section{B. Maximum likelihood based system identification and Generations of the binary excitation signals}

In this paper, the maximum likelihood based system identification method [3] is utilized. The frequency domain method is based on band-limited measurements of the input and the output signals, and can directly identify the sdomain transfer functions. Furthermore, it takes into consideration both input and output observation noise that present in measurements.

The input excitation is the key for good modeling results. For frequency domain analysis, the design goal for excitation signal is to put as much energy at the interesting frequencies as possible under the given restrictions. The generating method for binary excitation signals is discussed in [2]. A band-limited binary excitation sequence with designated frequency spectrum can be produced.

\section{Identification procedures}

The identification procedures for the whole identification process are the following,

1. Asymmetric friction component identification.

2. Poles and zeros of the lincar model identification.

3. The determinations for both friction magnitude and gain of the linear model.

The purpose of the first step is to find the asymmetric component of the friction. Compensating the asymmetric will balance the input signal entering the linear model, so as to make accurate identification for exact poles and zeros in the second step. By applying different excitation magnitudes, the friction magnitude and system gain can be evaluated by linear regression techniques.

\section{Experiment Results}

\section{A. Apparatus}

The precision table, Figure 1, is made by NSK Inc. It is driven by DC motors with low noise linear amplifiers. The specifications of the table are listed in tablel. The high 
precision table is cquipped with a $10 \mathrm{~mm}$ laser scalc manufactured by SONY. With the help of these measurement devices, the resolution of the tables has becn promoted to submicron level. Also, as a protection. two limit switches are mounted on two extreme points along the guide. The whole setup is established on a granite stone plate with vibration-resistance table.

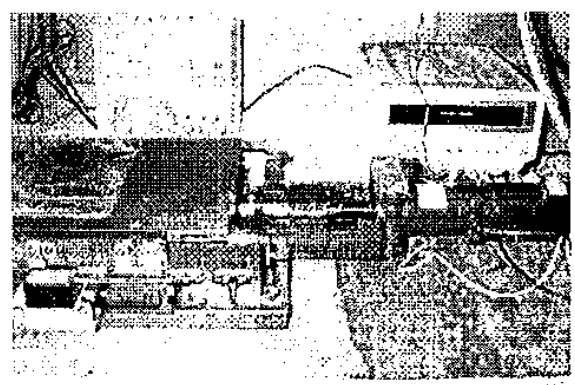

Figure 5. Precision Ballscrew Table with 10nmlaser scale.

\begin{tabular}{|c|c|}
\hline Table 1. Specifications of the table \\
\hline Stroke & $200 \mathrm{~mm}$ \\
\hline l'itch & $2 \mathrm{~mm}$ \\
\hline Repeatability & $0.001 \mathrm{~mm}$ \\
\hline Backlatsh & $0.001 \mathrm{~mm}$ \\
\hline Laser Seale Resolutiol & $10 \mathrm{~mm}$ \\
\hline
\end{tabular}

\section{B. Experimental Results}

In this section. the identification procedures are demonstrated by an cxample of the ballscrew table experiment. After properly choosing the closed-loop filter as

$$
\text { closed }- \text { loop filter }=\frac{5 s+50}{s} \text {, }
$$

The closed-loop system in Figure 4a is stabilized. The asymmetric friction component in the ballscrew table is found at $26 \mathrm{D} / \mathrm{A}$ counts, and that is about 0.127 Volt. In Figure 6, the input and ontput for the bias filter is draw at the upper graph. The lower graph shows the asymmetric friction component tracked.

A binary sequence is designed according to 12 , multiplying the $(-1,1)$ sequence with proper excitation magnitude and compensating for the asymmetric friction component, the excitation for identification is ready. After collecting the output data, the identification $\mathrm{IO}$ data is analyzed by maximum likelihood method $[3,4]$.
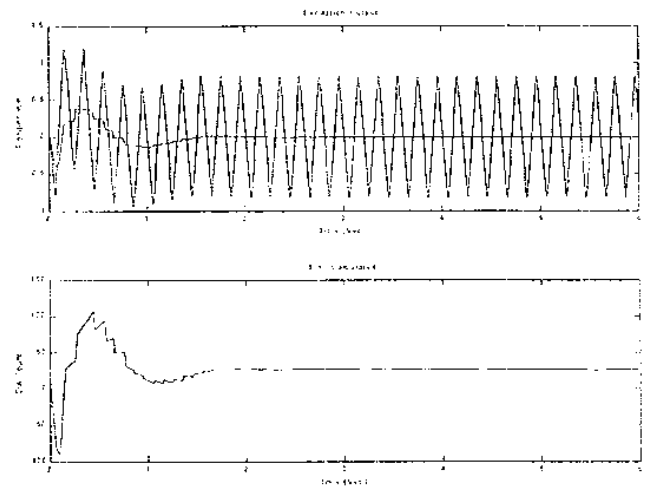

Figure 6. Bias filter input/output plot and asymmetric friction component tracked

The results are in Figure 7. By applying different excitation magnitudes, actually five magnitudes 580, 590, 600,610 and $620 \mathrm{D} / \mathrm{A}$ counts are tested in cxperiments. And the five identified pole sets are in Figure 8. The five pole sets are very similar cspccially at the low-frequency pole.
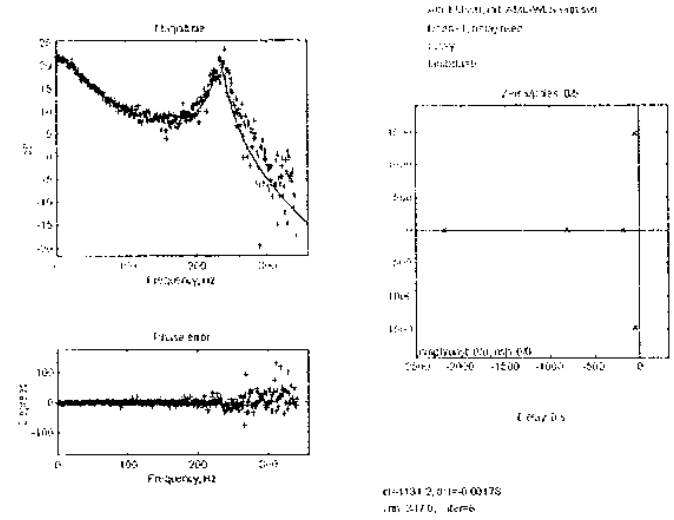

Figure 7 . The results of the fitted poles by maximumlikelihood

Bccause that the input binary sequence are different only at magnitude, by fixing the input data magnitude for all five evaluations, the calculated dc-gain will change proportional to magnitude changes. In Figure 9, by linear regression, a line can be draw with dc-gain calculated in $x-$ axis and applied magnitudes in $y$-axis. From this plot, the 
real dc-gain and the friction magnitudes can be evaluated. Finally, the system model is,

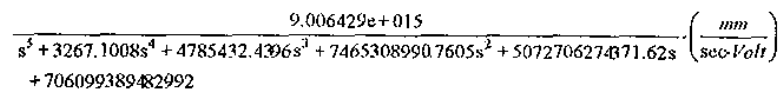

with Coulomb friction, 1.27volt in the plus direction and 1.14 in the minus direction. (Notice that the model in (9) is input voltage to system velocities).

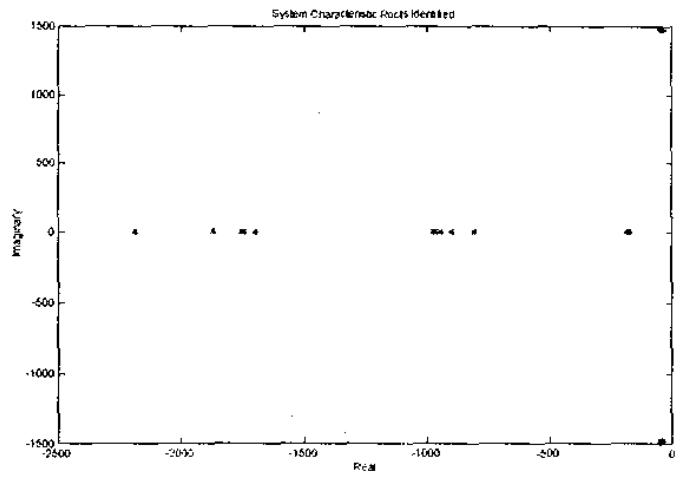

Figure 8 . The poles found by five different excitation magnitude $580,590,600,610$ and 620

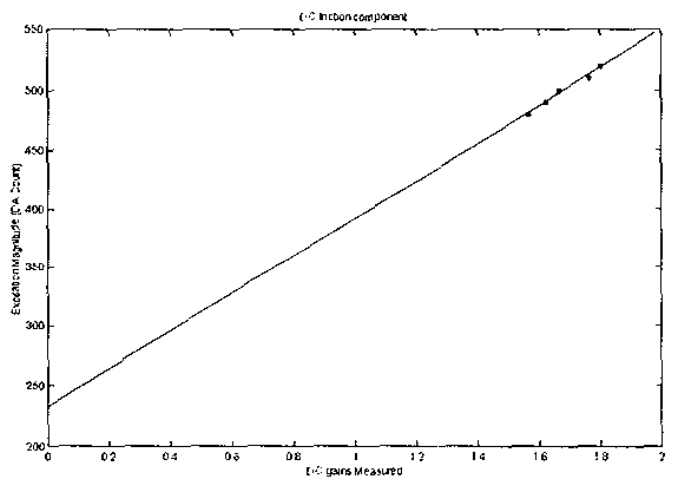

Figure 9. The magnitude of the friction (asymmetric canceled) can be found at dc-gain zero point. It is 234.089 in this case.

Figure 10 is the simulated model output and measured output, the identified model can keep up with the measured output. There are high frequency dynamics existed in the real plant, but in general, the dynamics of the ballscrew table in desired frequency span are faithfully identified.

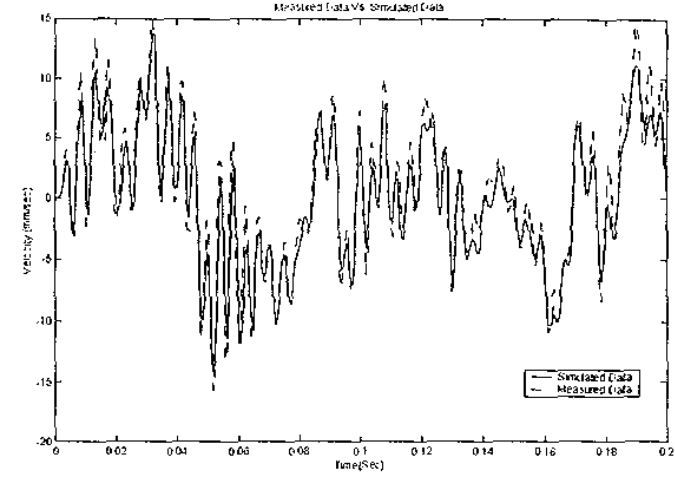

Figure 10. The measured output Vs identified model output.

\section{Conclusion}

By smartly choosing the excitation signal, the accuracy of the model can be increased even under friction nonlinearity. This idea can be extended to similar physical systems with the same nonlinear property. Or with proper modifications, it can be adapted to more complex situations in frequency response analysis.

\section{Reference}

[1] K. Ogata, Modern Control Lingineering, Prentice-Hall, 1990

[2] K.D. Paehlike and H. Rake,"Binary Multifrequency Signals-Synthesis and Application," Proc 5th IFAC Symposium on Identification and System Parameter Estimation, Vol. 1, pp. 589-596, 1979

[3] I. Kollar, "On Frequency Domain Identification of Linear Systems," IEEE Trans. On Instrumentation and Measurement, Vol. 42, No. 1, pp. 2-6, Feb. 1993

[4] I.Kollar, Matlab Frequency Domain System Identification Toolbox Manual, Math Works Inc, 1994.

[5] B. Armstrong-Helouvry, P. Dupont, and C. Canudas de Wit, "Survey of Models, Analysis Tools, and Compensation Methods for Control of Machines with Friction," Automatica, vol.30, no.7, pp. 1083-1138, July 1994. 